\title{
Electric Field-Driven On-Request Instant in Situ Formation/Removal of Solid Hydrogel within Microchannels for Efficient Electrophoretic Separation
}

Hongli Li,,$^{\dagger}$ Fengyun Li,,$^{\dagger}$ Lizhi Zhao, ${ }^{\dagger}$ Lei Zhao, ${ }^{\dagger}$ Ping Sun,,$^{\dagger}$ Jing Wu,,$^{\dagger}$ Xiayan Wang,,$^{\ddagger}$ Qiaosheng $P u^{*}, \dagger$

${ }^{\dagger}$ State Key Laboratory of Applied Organic Chemistry, Key Laboratory of Nonferrous Metals Chemistry and Resources Utilization of Gansu Province, Department of Chemistry, Lanzhou University, Lanzhou, Gansu, 730000, China.

${ }^{\star}$ Beijing Key Laboratory for Green Catalysis and Separation, Department of Chemistry and Chemical Engineering, Beijing University of Technology, Beijing, 100124, China.

Corresponding author:

Qiaosheng Pu

E-mail: puqs@1zu.edu.cn 


\section{The apparent EOF measurement.}

EOF was measured using a cross-type microchip with CS/ $\beta$-GP hydrogel in the separation channel by the neutral marker method. ${ }^{1} 500 \mathrm{nM}$ Rhodamine B was used as the marker (it is neutral between pH 6.0 and 10.8 ${ }^{2}$ ), $10 \mathrm{mM}$ borax with $40 \%$ methanol $(\mathrm{pH}$ 9.3) was used as the running buffer, a voltage of $2500 \mathrm{~V}$ was applied for separation. The EOF was calculated using the Eq.:

$$
\mu_{E O F}=\frac{L L_{e f f}}{V t}
$$

Here $L$ is the total length of the separation channel, $L_{\text {eff }}$ is the distance between injection and detection $(1.5 \mathrm{~cm}), \mathrm{V}$ is the applied voltage and $\mathrm{t}$ is the migration time of Rhodamine B. 

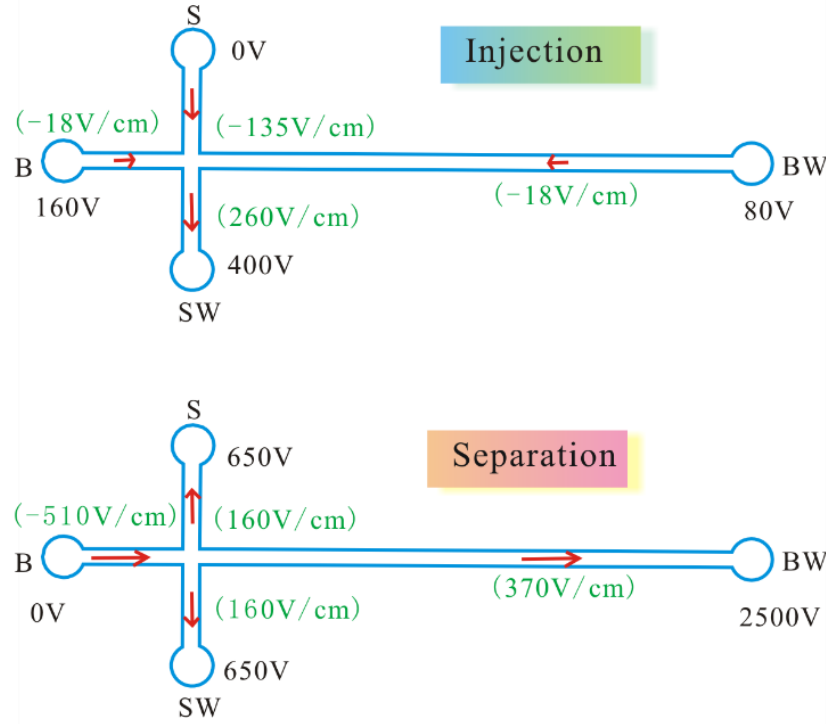

Figure S1. Schematic diagram of electric fields for sample injection and separation in microchip electrophoresis.
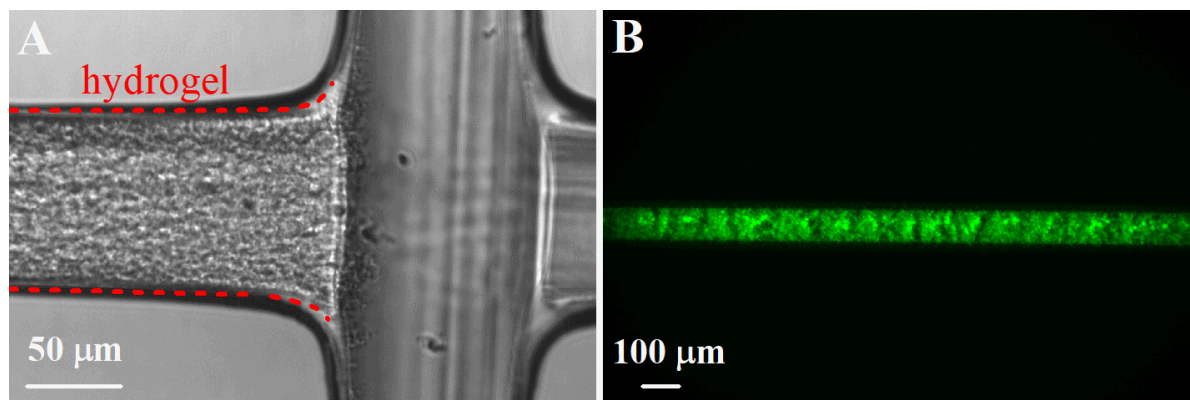

Figure S2. (A) Enlarged image of the interface of the CS/ $\beta$-GP hydrogel formed by electric field in the separation channel. (B) Fluorescent image of FITC-dyed CS/ $\beta$-GP hydrogel within the microchannel constructed by heating $\left(37^{\circ} \mathrm{C}\right)$ for $2 \mathrm{~h}$. 


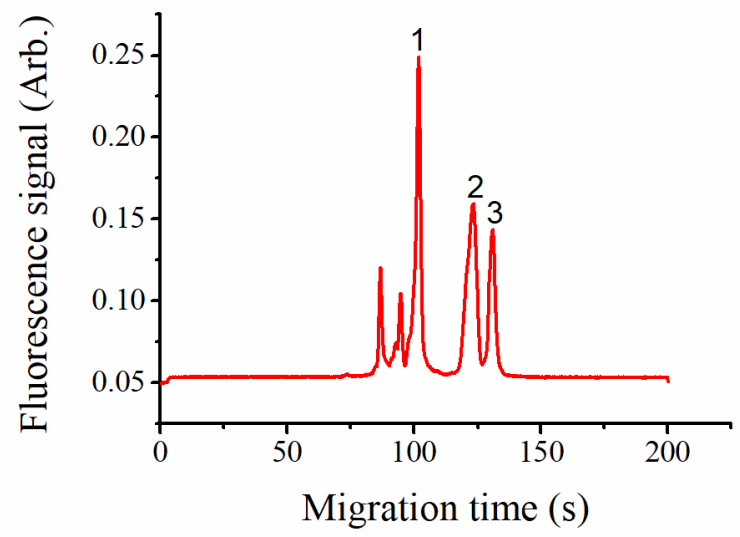

Figure S3. Electropherogram of FITC-labeled three peptides separated with CS/ $\beta$-GP hydrogel prepared by the electric field. Effective separation length: $2.5 \mathrm{~cm}$; inner diameter: $75 \mu \mathrm{m}$; separation voltage: 2500 V. Peaks: $1, \beta$-casomorphin (1-7), 2, gliadorphin 7, 3, $\beta$-casomorphin-7.

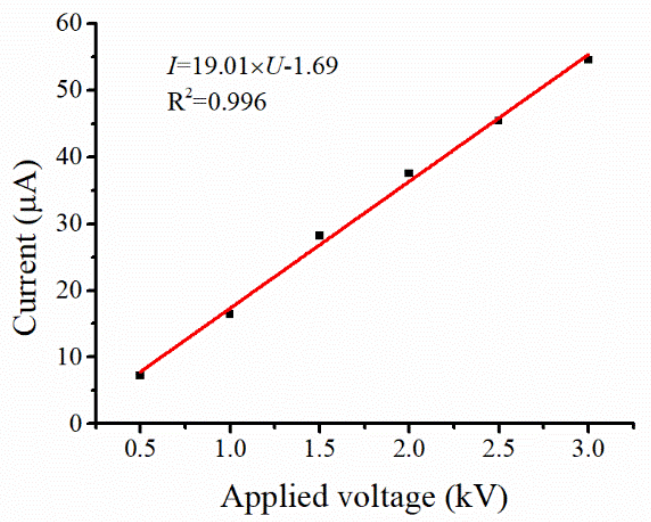

Figure S4. Relationship of current versus the applied voltage. Buffer: $10 \mathrm{mM}$ borax; the single straight channel length: $4 \mathrm{~cm}$. 


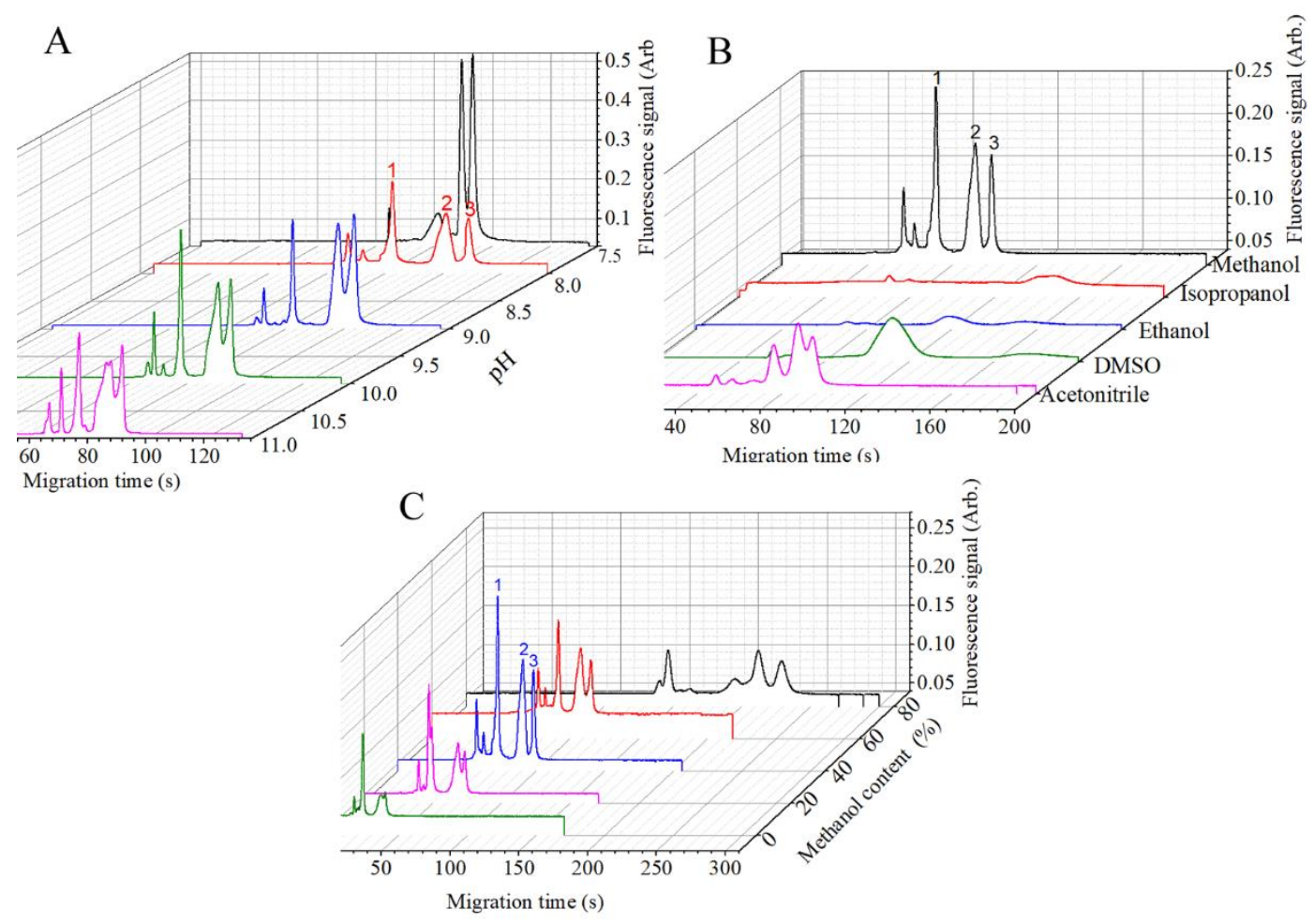

Figure S5. Effects of (A) pH, (B) 40\% (v/v) organic solvents, and (C) methanol concentration on the separation of FITC labeled three peptides. Effective separation length: $2.5 \mathrm{~cm}$; inner diameter: $75 \mu \mathrm{m}$; separation voltage: $2500 \mathrm{~V}$. Peaks: $1, \beta$-casomorphin $(1-7), 2$, gliadorphin 7,3 , $\beta$-casomorphin-7.
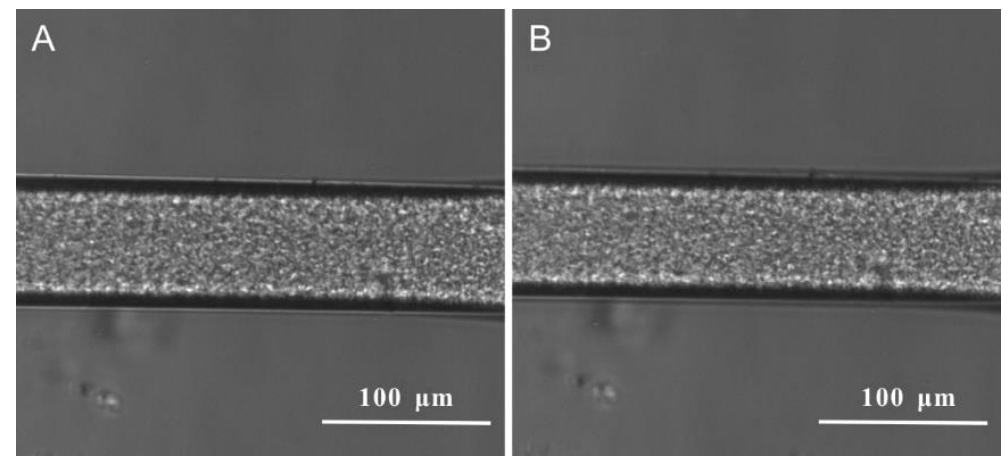

Figure S6. Microphotographs of the CS/ $\beta$-GP hydrogel (A) newly constructed by electric field, and (B) that after 200 runs of electrophoresis. 
Table S1. The intra-day, inter-day and chip-to-chip relative standard deviations (RSDs) of the migration times and peak areas of the main peaks of FITC labeled Hela cell lysate.

\begin{tabular}{|c|c|c|c|c|c|c|}
\hline \multirow{3}{*}{$\begin{array}{l}\text { Peak } \\
\text { No. }\end{array}$} & \multicolumn{2}{|c|}{ Intra-day $(\mathrm{n}=5)$} & \multicolumn{2}{|c|}{ Inter-day $(\mathrm{n}=5)$} & \multicolumn{2}{|c|}{ Chip-to-chip $(n=5)$} \\
\hline & Migration time & Peak area & Migration time & Peak area & Migration time & Peak area \\
\hline & $(\mathrm{RSD}, \%)$ & $(\mathrm{RSD}, \%)$ & $(\mathrm{RSD}, \%)$ & $(\mathrm{RSD}, \%)$ & $(\mathrm{RSD}, \%)$ & $(\mathrm{RSD}, \%)$ \\
\hline 1 & 0.11 & 0.71 & 1.3 & 1.5 & 2.5 & 2.7 \\
\hline 2 & 0.23 & 0.63 & 0.9 & 1.2 & 1.6 & 3.9 \\
\hline 3 & 0.25 & 0.72 & 1.0 & 2.1 & 3.6 & 2.9 \\
\hline 4 & 0.29 & 0.80 & 1.9 & 2.5 & 2.5 & 4.0 \\
\hline 5 & 0.17 & 0.58 & 2.2 & 2.0 & 3.6 & 3.9 \\
\hline
\end{tabular}

Table S2. Results for the determination of lactoferrin and transferrin in human serum samples.

\begin{tabular}{|c|c|c|c|c|c|c|c|}
\hline Sample & Analyte & $\begin{array}{l}\text { Content } \\
(\mu \mathrm{g} / \mathrm{mL})\end{array}$ & $\begin{array}{l}\text { Added } \\
(\mu \mathrm{g} / \mathrm{mL})\end{array}$ & $\begin{array}{l}\text { Totally found } \\
(\mu \mathrm{g} / \mathrm{mL})\end{array}$ & Recovery (\%) & $\begin{array}{l}\text { Intra-day } \\
\text { RSD (\%) } \\
(\mathrm{n}=5)\end{array}$ & $\begin{array}{l}\text { Inter-day } \\
\text { RSD (\%) } \\
(\mathrm{n}=3)\end{array}$ \\
\hline $1^{\mathrm{a}}$ & Lactoferrin & 0.16 & 0.20 & 0.36 & 100.3 & 2.9 & 2.2 \\
\hline \multirow{5}{*}{$2^{b}$} & \multirow{5}{*}{ Transferrin } & \multirow{5}{*}{2.5} & 0.40 & 0.52 & 90.0 & 1.8 & 3.6 \\
\hline & & & 1.00 & 1.18 & 101.9 & 2.6 & 1.4 \\
\hline & & & 1.5 & 3.98 & 98.7 & 4.1 & 3.5 \\
\hline & & & 3.0 & 5.39 & 96.4 & 2.7 & 1.9 \\
\hline & & & 6.0 & 8.79 & 104.9 & 3.5 & 3.2 \\
\hline
\end{tabular}




\section{REFERENCES}

(1) Wang, W.; Zhou, F.; Zhao, L.; Zhang, J. R.; Zhu, J. J. Measurement of Electroosmotic Flow in Capillary and Microchip Electrophoresis. J. Chromatogr. A 2007, $1170,1-8$.

(2) Schrum, K. F.; Lancaster, J. M.; Johnston, S. E.; Gilman, S. D. Monitoring Electroosmotic Flow by Periodic Photobleaching of a Dilute, Neutral Fluorophore. Anal. Chem. 2000, 72, 4317-4321. 